\title{
An approximate solution of a singular fractional telegraph equation by double Laplace decomposition method
}

\author{
Yahya T. Abdalla1, Hassan Eltayeb ${ }^{2}$ and Mohamed H. Khabir ${ }^{3}$ \\ ${ }^{1,3}$ Sudan University of Science and Technology, Faculty of Science, Department of Mathematics, Khartoum Sudan. \\ ${ }^{2}$ King Saud University, Faculty of Science, Department of Mathematics, Riyadh, Saudi Arabia.
}

Received: 15 August 2018, Accepted: 29 May 2019

Published online: 29 September 2019.

\begin{abstract}
In this artical, we introduce a robust method for solving a singular fractional telegraph equation, called Laplac transform decomposition method (DLDM).This method is combining between double Laplac transform method and the Adomian decomposition method . In this mthod,the fractional derivative is consider in Caputo sense. Some illustrative example to show that (DLDM) is rigorous and effective.
\end{abstract}

Keywords: Double Laplace transform, Adomian decomposition method, telegraph equation, Caputo fractional derivative and generalized Mittag-leffler function.

\section{Introduction}

Fractional partial differential equations are focus by scientists in different fields of science such as engineering, physics and mathematics. This focus comes from the significance and accuracy of fractional calculus. Many events in finance, signal analysis, control theory and biomedical science and other science can be described successfully by using fractional calculus. Telegraph equation is hyperbolic partial differential equation that is pertinent in several areas such as engineering, signal analysis and wave propagation [1]. In recent years, many authors convert telegraph equation to fractional telegraph equation and solved as linear, nonlinear, homogeneous and nonhomogeneous by different methods and given exact and approximate solution, in the literature authors used powerful methods, For example [2] using Laplace transform method,[3] suggested Laplace variational iteration method to obtained the approximate solution, the author [4] obtained the approximate solution of the telegraph equation by using double laplace transform method,[5] Homotopy perturbation technique,[6] radial basis functions and [7] introduced the Mixture of a new integral transform and homotopy perturbation method (HPM). Recently, the main objective of the present work is to provide the application of the double Laplace decomposition method to take out approximate solution of singular fractional telegraph equation (Bessel operator).

\section{Definitions}

In this section, we set up some basic definitions and preliminary used in this article.

Definition 1. [8] The Euler Gamma function $\Gamma(z)$ is defined by the integral:

$$
\Gamma(z)=\int_{0}^{\infty} e^{-t} t^{z-1} d t, \quad \mathbb{R}(z)>0 .
$$


Definition 2. [8] A two-parameter function of the Mittag-Leffler type is defined by the series expansion:

$$
E_{\alpha, \beta}(z)=\sum_{k=0}^{\infty} \frac{z^{k}}{\Gamma(\alpha k+\beta)}, \quad(\alpha>0, \beta>0) .
$$

Definition 3. [8] The Riemann-Lioville fractional integral operator $\rho>0$ of a function $f(t)$ is defined by:

$$
{ }_{a} D_{t}^{-\rho} f(t)=\frac{1}{\Gamma(\rho)} \int_{a}^{t}(t-\tau)^{\rho-1} f(\tau) d \tau
$$

Definition 4. [8] The left Riemann-Lioville fractional derivative is defined as:

$$
{ }_{a} D_{t}^{\rho} f(t)=\frac{1}{\Gamma(k-\rho)}\left(\frac{d}{d t}\right)^{k} \int_{a}^{t}(t-\tau)^{k-\rho-1} f(\tau) d \tau, \quad(k-1<\rho<k) .
$$

Definition 5. [8] The Right Riemann-Lioville fractional derivative is defined as:

$$
{ }_{t} D_{b}^{\rho} f(t)=\frac{1}{\Gamma(k-\rho)}\left(-\frac{d}{d t}\right)^{k} \int_{t}^{b}(t-\tau)^{k-\rho-1} f(\tau) d \tau, \quad(k-1<\rho<k) .
$$

Definition 6. [8] Caputo fractional derivative of order $\alpha>0$ of function an absolute continuous $f(t), t>0$ is defined as:

$$
{ }_{0} D_{t}^{\alpha} f(t)=\left\{\begin{array}{cc}
\frac{1}{\Gamma(\alpha-n)} \int_{0}^{t} \frac{f^{n}(\tau) d \tau}{(t-\tau)^{\alpha+1-n}}, & n-1<\alpha \leq n \in \mathbb{N} \\
\frac{d^{n}}{d t^{n}} f(t), & \alpha=n \in \mathbb{N}
\end{array}\right.
$$

where the function $f(t)$ has an absolute continuous derivative up to order $(n-1)$.

Definition 7. [9] The Laplace transform of a function $f(t)$ defined as:

$$
\mathfrak{L}[f(t)](s)=F(s)=\int_{0}^{\infty} e^{-s t} f(t) d t
$$

where $t>0$.

Definition 8. [8] The double Laplace transform for the partial fractional Caputo derivatives as:

$$
\mathfrak{L}_{x} \mathfrak{L}_{t}\left[\frac{\partial^{\alpha} u(x, t)}{\partial t^{\alpha}}\right]=s^{\alpha} U(p, s)-\sum_{k=0}^{n-1} s^{\alpha-k-1} \mathfrak{L}_{x}\left[\frac{\partial^{k} u(x, 0)}{\partial t^{k}}\right], \quad(n-1<\alpha \leq n) .
$$

Definition 9. [9,10] The inverse double Laplace transform $\mathfrak{L}_{p}^{-1} \mathfrak{L}_{s}^{-1}[F(p, s)]=f(x, t)$ is defined by the complex double integral formula:

$$
\mathfrak{L}_{x}^{-1} \mathfrak{L}_{t}^{-1}[F(p, s)]=f(x, t)=\frac{1}{2 \pi i} \int_{c-i \infty}^{c+i \infty} e^{p x} d p \frac{1}{2 \pi i} \int_{d-i \infty}^{d+i \infty} e^{s t} F(p, s) d s,
$$

where $F(p, s)$ must be an analytic function for all $p$ and $s$ in the region defined by the inequalities $\mathfrak{R e}(p) \geq c$ and $\mathfrak{R e}(s)$ $\geq d$, where $c$ and $d$ are real constants to be chosen suitably.

Definition 10. [11] The Adomian Decomposition Method is defined as:

$$
A_{n}=\frac{1}{n !} \frac{d^{n}}{d \lambda^{n}}\left[G\left(\sum_{i=0}^{\infty} \zeta_{i} \lambda^{i}\right)\right]_{\lambda=0}
$$




\section{Proposed fractional double Laplace decomposition method (FDLDM)}

In this section, we derive the main idea of fractional double laplace decomposition method to solve a singular (Bessel operator) fractional telegraph equation.

Theorem 1. We consider singular fractional telegraph equation with boundary condition as follows:

$$
D_{x}^{2 \alpha} u(x, t)=\frac{1}{t}\left(t D_{t} u(x, t)\right)_{t}+u(x, t)+h(x, t), 0<\alpha \leq 1 \text { and } x, t \geq 0
$$

Subject to boundary conditions,

$$
u(0, t)=f_{1}(t) \text { and } u_{x}(0, t)=f_{2}(t)
$$

where $\frac{1}{t}\left(t D_{t} u(x, t)\right)_{t}$ is the Bessel operator and $h(x, t)$ is a continuous function.

Then the solution of Eq.(11) is given by

$$
\begin{aligned}
& u_{0}(x, t)=G(x, t)=\frac{1}{p^{2 \alpha}} H(x, t)+\frac{1}{p} F_{1}(s)+\frac{1}{p^{2}} F_{2}(s) \\
& u_{n+1}(x, t)=\mathfrak{L}_{x}^{-1} \mathfrak{L}_{t}^{-1}\left[\frac{1}{p^{2 \alpha}} \mathfrak{L}_{x} \mathfrak{L}_{t}\left[D_{t t} u_{n}(x, t)+D_{t} u_{n}(x, t)+u_{n}(x, t)\right]\right], \quad n \geq 0
\end{aligned}
$$

Proof. In order to obtain the solution of Eq.(11), we apply the fractional double Laplace transform of partial derivatives for equation Eq.(11), we get

$$
p^{2 \alpha} U(p, s)-p^{2 \alpha-1} U(0, s)-p^{2 \alpha-2} U_{x}(0, s)=\mathfrak{L}_{x} \mathfrak{L}_{t}\left[D_{t t} u_{n}(x, t)+\frac{1}{t} D_{t} u_{n}(x, t)+u(x, t)+h(x, t)\right]
$$

using the single Laplace transform for boundaryl conditions Eq.(12), we have,

$$
\begin{gathered}
\mathfrak{L}_{t}[u(0, t)]=\mathfrak{L}_{t}\left[f_{1}(t)\right]=F_{1}(s) \\
\mathfrak{L}_{t}\left[u_{x}(0, t)\right]=\mathfrak{L}_{t}\left[f_{2}(t)\right]=F_{2}(s),
\end{gathered}
$$

by substituting Eq.(15) in Eq.(14), use the property of the double Laplace transform and simplifying, we obtain

$$
U(p, s)=\frac{1}{p^{2 \alpha}} \mathfrak{L}_{x} \mathfrak{L}_{t}\left[D_{t t} u(x, t)+\frac{1}{t} D_{t} u(x, t)+u_{n}(x, t)\right]+\frac{1}{p^{2 \alpha}} H(x, t)+\frac{1}{p} F_{1}(s)+\frac{1}{p^{2}} F_{2}(s),
$$

where $H(x, t)$ is double Laplace of $h(x, t)$. Taking inverse double Laplace transform to Eq.(16), we get,

$$
u(x, t)=G(x, t)+\mathfrak{L}_{x}^{-1} \mathfrak{L}_{t}^{-1}\left[\frac{1}{p^{2 \alpha}} \mathfrak{L}_{x} \mathfrak{L}_{t}\left[D_{t t} u(x, t)+\frac{1}{t} D_{t} u(x, t)+u_{n}(x, t)\right]\right],
$$

where $G(x, t)$ is the function comes from continuous function and initial condition. The solution of Eq.(17) can be written as infinite series terms (Adomian decomposition method) such as

$$
u(x, t)=\sum_{n=0}^{\infty} u_{n}(x, t)
$$

then Eq.(17) become

$$
\sum_{n=0}^{\infty} u_{n}(x, t)=G(x, t)+\mathfrak{L}_{x}^{-1} \mathfrak{L}_{t}^{-1}\left[\frac{1}{p^{2 \alpha}} \mathfrak{L}_{x} \mathfrak{L}_{t}\left[\sum_{n=0}^{\infty} D_{t t} u_{n}(x, t)+\frac{1}{t} \sum_{n=0}^{\infty} D_{t} u_{n}(x, t)+\sum_{n=0}^{\infty} u_{n}(x, t)\right]\right]
$$


Eq.(19) can be written as

$$
u_{0}+u_{1}+u_{2}+\ldots=G(x, t)+\mathfrak{L}_{x}^{-1} \mathfrak{L}_{t}^{-1}\left[\frac{1}{p^{2 \alpha}} \mathfrak{L}_{x} \mathfrak{L}_{t}\left[u_{0 t t}+u_{1 t t}+\ldots\right]+\frac{1}{t}\left[u_{0 t}+u_{1 t}+\ldots\right]+\left[u_{0}+u_{1}+\ldots\right]\right]
$$

the method suggests that the zero component $u_{0}(x, t)$ is identified by terms that are not included under $\mathfrak{L}_{x}^{-1} \mathfrak{L}_{t}^{-1}$ in Eq.(20).

$$
\begin{aligned}
& u_{0}(x, t)=G(x, t)=\frac{1}{p^{2 \alpha}} H(x, t)+\frac{1}{p} F_{1}(s)+\frac{1}{p^{2}} F_{2}(s) \\
& u_{n+1}(x, t)=\mathfrak{L}_{x}^{-1} \mathfrak{L}_{t}^{-1}\left[\frac{1}{p^{2 \alpha}} \mathfrak{L}_{x} \mathfrak{L}_{t}\left[u_{n t t}+\frac{1}{t} u_{n t}+u_{n}(x, t)\right]\right] . n \geq 0
\end{aligned}
$$

Note that the inverse double Laplace transform of each terms in the right side of Eq.(21) exists.

\section{Numrical examples}

In this section, we demonstrate the applicability and stability of our method by applying numerical examples

Example 1. We consider the following singular fractional telegraph equation:

$$
\begin{gathered}
D_{x}^{2 \alpha} u(x, t)=\frac{1}{t}\left(t D_{t} u(x, t)\right)_{t}+u+2 t^{2}-4 x^{2}-x^{2} t^{2}, \\
x, t \geq 0 \text { and } 0<\alpha \leq 1
\end{gathered}
$$

Subject to the initial condition:

$$
u(0, t)=0, \quad u_{x}(0, t)=0
$$

\section{Solution 1.}

Appling the double Laplace transform on both sides of Eq.(22) to get,

$$
p^{2 \alpha} U(p, s)-p^{2 \alpha-1} U(0, s)-p^{2 \alpha-2} U_{x}(0, s)=2 \frac{2 !}{p s^{3}}-4 \frac{2 !}{p^{3} s}-\frac{2 ! 2 !}{p^{3} s^{3}}+\mathfrak{L}_{x} \mathfrak{L}_{t}\left[D_{t t} u(x, t)+\frac{1}{t} D_{t} u(x, t)+u_{n}(x, t)\right]
$$

taking single Laplace transform to Eq.(23) and substitute in Eq.(24) gives,

$$
U(p, s)=2 \frac{2 !}{p^{2 \alpha+1} s^{3}}-4 \frac{2 !}{p^{2 \alpha+3} s}-\frac{2 ! 2 !}{p^{2 \alpha+3} s^{3}}+\frac{1}{p^{2 \alpha}} \mathfrak{L}_{x} \mathfrak{L}_{t}\left[D_{t t} u(x, t)+\frac{1}{t} D_{t} u(x, t)+u_{n}(x, t)\right],
$$

using the inverse double Laplace transform for Eq.(25) we have,

$$
u(x, t)=2 \frac{x^{2 \alpha}}{\Gamma(2 \alpha+1)} t^{2}-8 \frac{x^{2 \alpha+2}}{\Gamma(2 \alpha+3)}-2 \frac{x^{2 \alpha+2}}{\Gamma(2 \alpha+3)} t^{2}+\mathfrak{L}_{x}^{-1} \mathfrak{L}_{t}^{-1}\left[\frac{1}{p^{2 \alpha}} \mathfrak{L}_{x} \mathfrak{L}_{t}\left[D_{t t} u(x, t)+\frac{1}{t} D_{t} u(x, t)+u_{n}(x, t)\right]\right],
$$

now we define the function $u(x, t)$ by the decomposition series,

$$
u(x, t)=\sum_{n=0}^{\infty} u_{n}(x, t),
$$

insert Eq.(27) into both sides Eq.(26) gives

$$
\begin{aligned}
\sum_{n=0}^{\infty} u_{n}(x, t)=2 \frac{x^{2 \alpha}}{\Gamma(2 \alpha+1)} t^{2}-8 \frac{x^{2 \alpha+2}}{\Gamma(2 \alpha+3)}-2 \frac{x^{2 \alpha+2}}{\Gamma(2 \alpha+3)} t^{2} \\
+\mathfrak{L}_{x}^{-1} \mathfrak{L}_{t}^{-1}\left[\frac{1}{p^{2 \alpha}} \mathfrak{L}_{x} \mathfrak{L}_{t}\left[\sum_{n=0}^{\infty} u_{n t t}(x, t)+\frac{1}{t} \sum_{n=0}^{\infty} u_{n t}(x, t)+\sum_{n=0}^{\infty} u_{n}(x, t)\right]\right],
\end{aligned}
$$


by using the Eq.(28) we find a few terms of the series of $u(x, t)$ as,

$$
\begin{aligned}
& u_{0}(x, t)=2 \frac{x^{2 \alpha}}{\Gamma(2 \alpha+1)} t^{2}-8 \frac{x^{2 \alpha+2}}{\Gamma(2 \alpha+3)}-2 \frac{x^{2 \alpha+2}}{\Gamma(2 \alpha+3)} t^{2}, \\
& u_{n+1}(x, t)=\mathfrak{L}_{x}^{-1} \mathfrak{L}_{t}^{-1}\left[\frac{1}{p^{2 \alpha}} \mathfrak{L}_{x} \mathfrak{L}_{t}\left[\sum_{n=0}^{\infty} u_{n t t}(x, t)+\frac{1}{t} \sum_{n=0}^{\infty} u_{n t}(x, t)+\sum_{n=0}^{\infty} u_{n}(x, t)\right]\right], \quad n \geq 0,
\end{aligned}
$$

then

$$
\begin{aligned}
& u_{1}(x, t)=8 \frac{x^{4 \alpha}}{\Gamma(4 \alpha+1)}-16 \frac{x^{4 \alpha+2}}{\Gamma(4 \alpha+3)}+2 \frac{x^{4 \alpha}}{\Gamma(4 \alpha+1)} t^{2}-2 \frac{x^{4 \alpha+2}}{\Gamma(4 \alpha+3)} t^{2}, \\
& u_{2}(x, t)=16 \frac{x^{6 \alpha}}{\Gamma(6 \alpha+1)}-24 \frac{x^{6 \alpha+2}}{\Gamma(6 \alpha+3)}+2 \frac{x^{6 \alpha}}{\Gamma(6 \alpha+1)} t^{2}-2 \frac{x^{6 \alpha+2}}{\Gamma(6 \alpha+3)} t^{2},
\end{aligned}
$$

therefore, the (FDLDM) give us the series solution of Eq.(22) is,

$$
\begin{aligned}
u(x, t)= & u_{0}(x, t)+u_{1}(x, t)+u_{2}(x, t)+u_{3}(x, t)+\ldots \\
= & 2 \frac{x^{2 \alpha}}{\Gamma(2 \alpha+1)} t^{2}-8 \frac{x^{2 \alpha+2}}{\Gamma(2 \alpha+3)}-2 \frac{x^{2 \alpha+2}}{\Gamma(2 \alpha+3)} t^{2}+8 \frac{x^{4 \alpha}}{\Gamma(4 \alpha+1)}-16 \frac{x^{4 \alpha+2}}{\Gamma(4 \alpha+3)}+2 \frac{x^{4 \alpha}}{\Gamma(4 \alpha+1)} t^{2} \\
& -2 \frac{x^{4 \alpha+2}}{\Gamma(4 \alpha+3)} t^{2}+16 \frac{x^{6 \alpha}}{\Gamma(6 \alpha+1)}-24 \frac{x^{6 \alpha+2}}{\Gamma(6 \alpha+3)}+2 \frac{x^{6 \alpha}}{\Gamma(6 \alpha+1)} t^{2}-2 \frac{x^{6 \alpha+2}}{\Gamma(6 \alpha+3)} t^{2}+\ldots .
\end{aligned}
$$

If we take $(\alpha=1)$ then we get exact solution of standard telegraph equation as,

$$
u(x, t)=x^{2} t^{2}
$$

Example 2. Consider the following singular fractional telegraph equation:

$$
\begin{gathered}
D_{x}^{2 \alpha} u(x, t)=\frac{1}{t}\left(t D_{t} u(x, t)\right)_{t}+u-x \ln (t), \\
x, t \geq 0 \text { and } 0<\alpha \leq 1
\end{gathered}
$$

Subject to the initial condition:

$$
u(0, t)=0, \quad u_{x}(0, t)=\ln (t) .
$$

\section{Solution 2.}

Appling double Laplace transform for Eq.(34) we get,

$$
p^{2 \alpha} U(p, s)-p^{2 \alpha-1} U(0, s)-p^{2 \alpha-2} U_{x}(0, s)=\frac{\ln (s)+\gamma}{p^{2} s}+\mathfrak{L}_{x} \mathfrak{L}_{t}\left[D_{t t} u(x, t)+\frac{1}{t} D_{t} u(x, t)+u_{n}(x, t)\right],
$$

taking single Laplace transform Eq.(35) and substitute in Eq.(36) gives,

$$
U(p, s)=\frac{\ln (s)+\gamma}{p^{2} s}+\frac{\ln (s)+\gamma}{p^{2 \alpha+2} s}+\frac{1}{p^{2 \alpha}} \mathfrak{L}_{x} \mathfrak{L}_{t}\left[D_{t t} u(x, t)+\frac{1}{t} D_{t} u(x, t)+u_{n}(x, t)\right],
$$

using the inverse double laplace transform transform for Eq.(37) we have,

$$
u(x, t)=x \ln (t)+\frac{x^{2 \alpha+1}}{\Gamma(2 \alpha+2)} \ln (t)+\mathfrak{L}_{x}^{-1} \mathfrak{L}_{t}^{-1}\left[\frac{1}{p^{2 \alpha}} \mathfrak{L}_{x} \mathfrak{L}_{t}\left[D_{t t} u(x, t)+\frac{1}{t} D_{t} u(x, t)+u_{n}(x, t)\right]\right],
$$


now we define the function $u(x, t)$ by the decomposition series,

$$
u(x, t)=\sum_{n=0}^{\infty} u_{n}(x, t)
$$

insert Eq.(39) into both sides of Eq.(38) gives,

$$
\sum_{n=0}^{\infty} u_{n}(x, t)=x \ln (t)-\frac{x^{2 \alpha+1}}{\Gamma(2 \alpha+2)} \ln (t)+\mathfrak{L}_{x}^{-1} \mathfrak{L}_{t}^{-1}\left[\frac{1}{p^{2 \alpha}} \mathfrak{L}_{x} \mathfrak{L}_{t}\left[\sum_{n=0}^{\infty} D_{t t} u_{n}(x, t)+\frac{1}{t} \sum_{n=0}^{\infty} D_{t} u_{n}(x, t)+\sum_{n=0}^{\infty} u_{n}(x, t)\right]\right]
$$

by using Eq.(40) we find a few terms of the series of $u(x, t)$,

$$
\begin{gathered}
u_{0}=x \ln (t)-\frac{x^{2 \alpha+1}}{\Gamma(2 \alpha+2)} \ln (t) \\
u_{n+1}(x, t)=\mathfrak{L}_{x}^{-1} \mathfrak{L}_{t}^{-1}\left[\frac{1}{p^{2 \alpha}} \mathfrak{L}_{x} \mathfrak{L}_{t}\left[D_{t t} u_{n}(x, t)+\frac{1}{t} D_{t} u_{n}(x, t)+u_{n}(x, t)\right]\right], \quad n \geq 0, \\
u_{1}(x, t)=\frac{x^{2 \alpha+1}}{\Gamma(2 \alpha+2)} \ln (t)-\frac{x^{2 \alpha+3}}{\Gamma(2 \alpha+4)} \ln (t), \\
u_{2}(x, t)=\frac{x^{2 \alpha+3}}{\Gamma(2 \alpha+3)} \ln (t)-\frac{x^{2 \alpha+5}}{\Gamma(2 \alpha+6)} \ln (t), \\
u_{3}(x, t)=\frac{x^{2 \alpha+5}}{\Gamma(2 \alpha+5)} \ln (t)-\frac{x^{2 \alpha+7}}{\Gamma(2 \alpha+8)} \ln (t) .
\end{gathered}
$$

therefore, the (FDLDM) give us the series solution of Eq.(34) is,

$$
\begin{aligned}
u(x, t)= & u_{0}(x, t)+u_{1}(x, t)+u_{2}(x, t)+u_{3}(x, t)+\ldots \\
u(x, t)= & x \ln (t)-\frac{x^{2 \alpha+1}}{\Gamma(2 \alpha+2)} \ln (t)+\frac{x^{2 \alpha+1}}{\Gamma(2 \alpha+2)} \ln (t)-\frac{x^{2 \alpha+3}}{\Gamma(2 \alpha+4)} \ln (t)+\frac{x^{2 \alpha+3}}{\Gamma(2 \alpha+3)} \ln (t) \\
& -\frac{x^{2 \alpha+5}}{\Gamma(2 \alpha+6)} \ln (t)+\frac{x^{2 \alpha+5}}{\Gamma(2 \alpha+5)} \ln (t)-\frac{x^{2 \alpha+7}}{\Gamma(2 \alpha+8)} \ln (t)+\ldots .
\end{aligned}
$$

The solution of Eq.(34) is equal to the exact solution of standard telegraph equation ( when $\alpha=1$ ):

$$
u(x, t)=\ln \left(t^{x}\right)
$$

\section{Numrical results}

In this section, we shall illustrated the accuracy and efficiency of the fractional double Laplace decomposition method (FDLDM).Figure (1) discuss the exact solution and approximate solution of example (1) we get infinitesimal error equal $\left(2.775557561562891 e^{-17}\right)$ that means the present method is forceful and accurately method.Figure (2) approximate solution of example (1) the behavior of the function with various values of fractional $\alpha=0.94,0.96,0.98$ and 1 , we see that the function $u(x, t)$ increasing when $\alpha$ is decreasing with increasing the $x$ at the value of $t=1$. Table (1), tells us the absolute error for example (1) by compering the exact solution and approximate solution $u_{8}$ obtained by the (FDLDM) at $\alpha=1$ and different values of $t$. In example (2). The exact solution and approximate solution are equal $\ln \left(t^{x}\right)$ by canceling the noise terms, notice that the solution is verified in Eq.(34) when $\alpha=1$. 


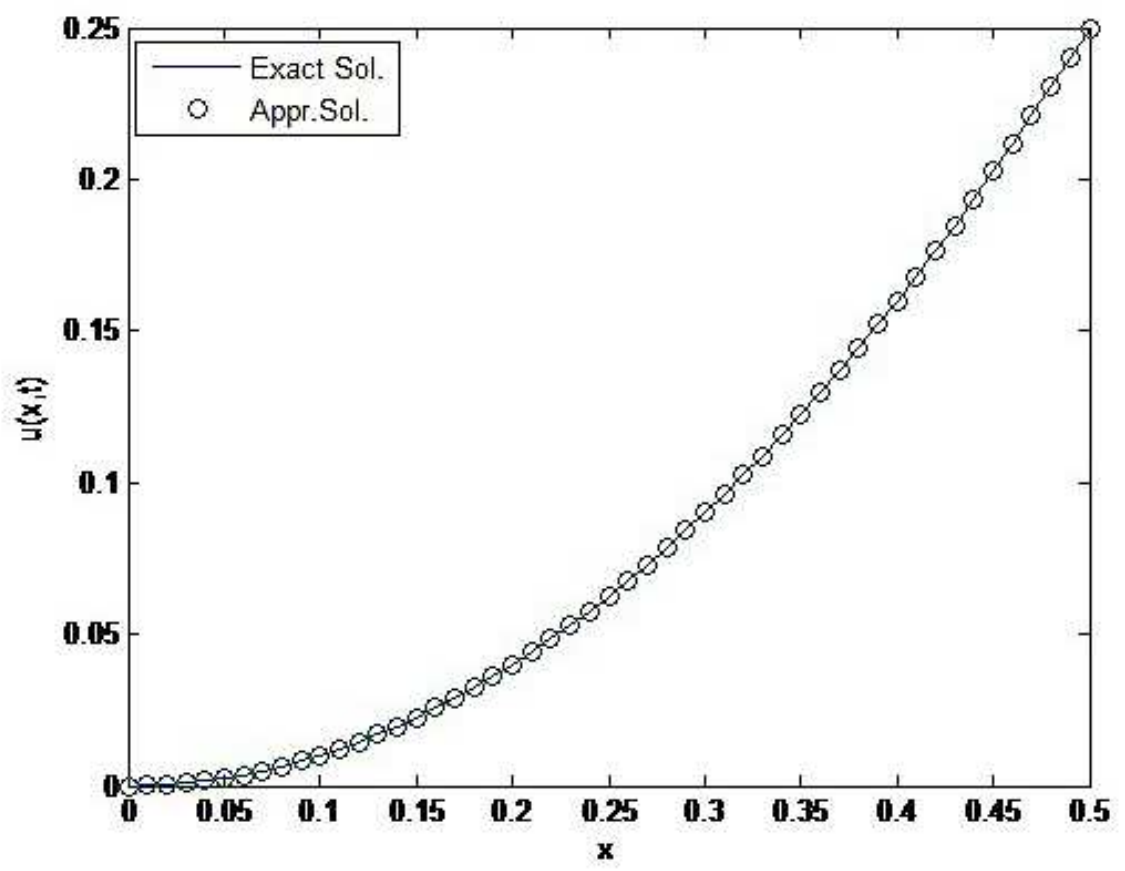

Fig. 1: The graph of Exact and approximate solutions of $u(x, t)$ for Example 1 .

\begin{tabular}{|l|l|l|l|}
\hline $\mathrm{t}$ & Exact Solution & $\begin{array}{l}\text { Approximate Solution } \\
\alpha=1\end{array}$ & $\begin{array}{l}\mathrm{E}_{8}(u)=\left|u-u_{8}\right| \\
\text { for } \alpha=1\end{array}$ \\
\hline 0.2 & 0.010000000000000 & 0.010000000000000 & $1.734723475976807 \mathrm{e}-18$ \\
\hline 0.4 & 0.040000000000000 & 0.040000000000000 & 0 \\
\hline 0.6 & 0.090000000000000 & 0.090000000000000 & $1.387778780781446 \mathrm{e}-17$ \\
\hline 0.8 & 0.160000000000000 & 0.160000000000000 & $2.775557561562891 \mathrm{e}-17$ \\
\hline 1.0 & 0.250000000000000 & 0.250000000000000 & $2.775557561562891 \mathrm{e}-17$ \\
\hline
\end{tabular}

Table 1: Exact Solution and Approximate Solution of $u(x, t)$ for Example 1 with $n=51$ at $\alpha=1$.

\section{Conclusion}

We have successfully applied double Laplace transform and adomian decomposition method to obtain the approximate solutions of the fractional telegraph equation.The (FDLDM) give us small error and high convergence. As seen in Table 1 , this techniques lead us to say the mathod has high accurate and efficient solutions.

\section{Competing interests}

The authors declare that they have no competing interests.

\section{Authors' contributions}

All authors have contributed to all parts of the article. All authors read and approved the final manuscript. 


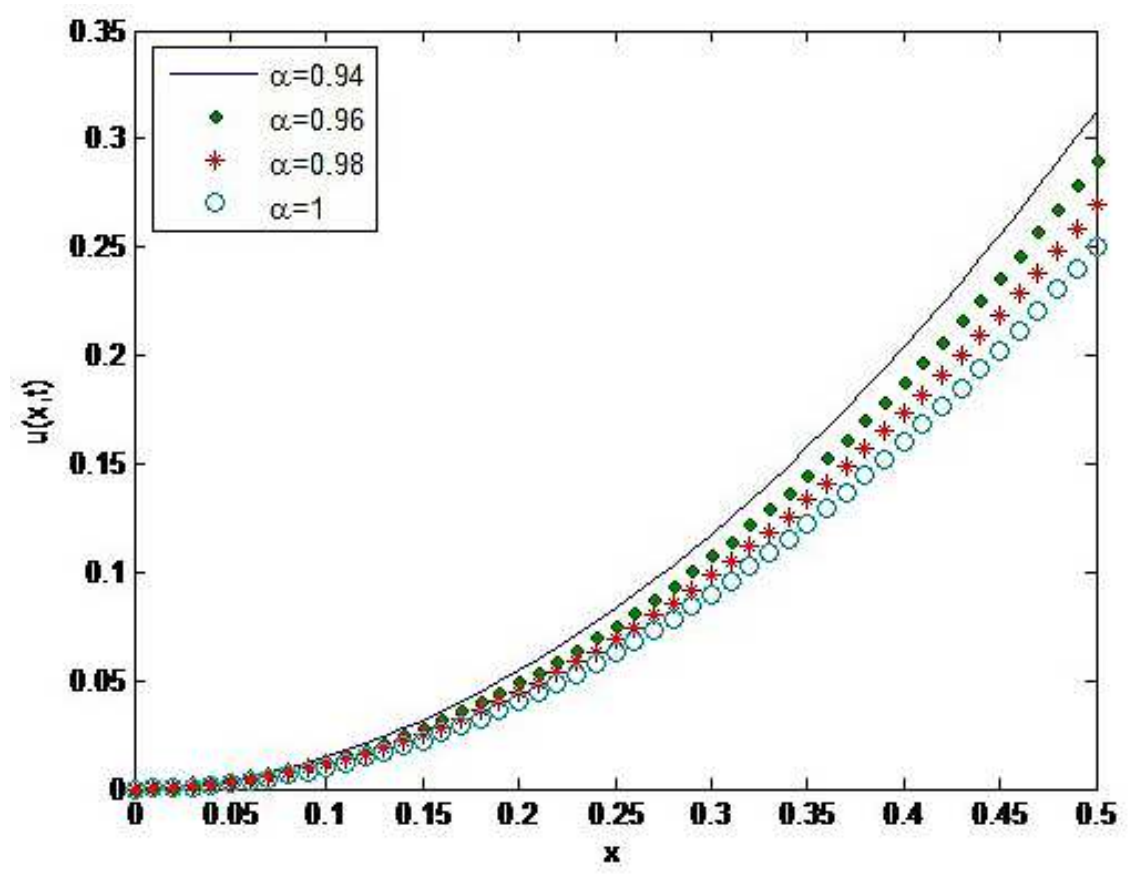

Fig. 2: The approximate solutions of $\mathrm{u}(\mathrm{x}, \mathrm{t})$ for Example 1 for $\alpha=0.94,0.96,0.98$, and 1 .

\section{References}

[1] N. Sweilam, A. Nagy and A. A. El-Sayed, Solving time-fractional order telegraph equation via sinc-legendre collocation method, Mediterranean Journal of Mathematics 13 (2016), no. 6, 5119-5133.

[2] S. Kumar, A new analytical modelling for fractional telegraph equation via laplace transform, Applied Mathematical Modelling 38 (2014), no. 13, 3154-3163.

[3] F. A. Alawad, E. A. Yousif and A. I. Arbab, A new technique of laplace variational iteration method for solving space-time fractional telegraph equations, International Journal of Differential Equations 2013 (2013).

[4] R. R. Dhunde and G. Waghmare, Double laplace transform combined with iterative method for solving non-linear telegraph equation, Journal of the Indian Mathematical Society 83 (2016), no. 3-4, 221-230.

[5] U. Hayat and S. Mohyud-Din, Homotopy perturbation technique for time fractional telegraph equations, International Journal of Modern Theoretical Physics 2 (2013), no. 1, 33-41.

[6] V. R. Hosseini, W. Chen and Z. Avazzadeh, Numerical solution of fractional telegraph equation by using radial basis functions, Engineering Analysis with Boundary Elements 38 (2014), 31-39.

[7] A. Kashuri, A. Fundo and M. Kreku, Mixture of a new integral transform and homotopy perturbation method for solving nonlinear partial differential equations, Advances in Pure Mathematics 3 (2013), no.03, 317.

[8] I. Podlubny, Fractional differential equations: An introduction to fractional derivatives, fractional differential equations, to methods of their solution and some of their applications, vol. 198, Elsevier, 1998.

[9] H. Eltayeb and A. Kiliçman, A note on solutions of wave, laplace's and heat equations with convolution terms by using a double laplace transform, Applied Mathematics Letters 21 (2008), no. 12, 1324-1329.

[10] A. Kılıçman and H. Eltayeb, A note on defining singular integral as distribution and partial differential equations with convolution term, Mathematical and Computer Modelling 49 (2009), no. 1-2, 327-336.

[11] A.-M. Wazwaz, Partial differential equations and solitary waves theory, Springer Science \& Business Media, 2010. 